\title{
WHO SHOULD ASK? Ethical Interviewing in Psychiatric Epidemiology Studies
}

Joanne E. Turnbull, Ph.D., Jane D. McLeod, Ph.D., Jay M. Callahan, M.S.W., M.A., Ronald C. Kessler, Ph.D.

In a recent survey examining responses to life stress, difficulties were encountered by lay interviewers. These are addressed, as are ethical issues arising from the combination of survey and clinical methodologies. The issues of respondent harm and informed consent are discussed and initial strategies outlined.

$E^{\mathrm{p}}$ pidemiology comprises the study of the physical and social environments as they relate to the distribution of disease in human populations. Epidemiologic methods serve three main purposes: to identify cases of disease; to examine patterns of disease occurrence; and to trace the historical determinants of these patterns (Last, 1983). On a theoretical level, epidemiology seeks to understand the association between a disease and specific population characteristics in the hope of identifying the origin of the disease. On a practical level, it contributes to prevention programs like those that eradicated or mitigated malaria, smallpox, and poliomyelitis (Tancredi, 1986).

Traditionally, epidemiologic methods have been applied to physical disease, playing only a minor role in the study of mental disorders. With notable exceptions (Dunham, 1965; Faris \& Dunham, 1939; Mischler \& Scotch, 1963), psychiatric re- search has been confined to clinical settings and to study samples comprised of treated psychiatric patients. In recent years, however, psychiatric epidemiology has emerged as a new epidemiologic subspecialty which focuses on the etiology of, and risk factors associated with, mental disorders in the general population. Most of the studies conducted under this new subspecialty employ survey research methods to study the relationship between social stress and mental health.

These survey studies combine the traditional epidemiologic interest in the incidence and prevalence of specific psychiatric disorders with sophisticated design and analysis techniques. By going beyond the boundaries of the treatment setting and into the community, survey strategies promise to yield valuable insights into the relationship between stress and the onset of serious mental health problems. They open several

Based on a paper presented at the 1986 annual meeting of the American Orthopsychiatric Association in Chicago. Research was supported in part by NIMH grant 63501 and Training Grant No. T32 MHI 6806-03. Authors are at: Duke University Medical Center, Durham, NC (Turnbull); State University of New York, Albany (McLeod); and University of Michigan, Ann Arbor (Callahan, Kessler). 
lines of inquiry that are not feasible in treated samples. For example, true prevalence rates for specific disorders can only be estimated accurately from general population samples, since treated samples do not include persons who have the disorder but who are not under treatment. In addition, general population studies allow the barriers to care, as well as informal helping networks of those who do not use formal sources of help, to be explored. Risk factors for the development of mental disorders can also be specified if individuals are studied before entering treatment. All of this information is crucial to prevention efforts for specific psychiatric disorders.

Despite these important benefits, complex issues and new challenges arise when survey research strategies are employed to collect clinical information for the study of psychiatric vulnerability in the general population. Survey research then enters into the province of the mental health professional. This shift creates both practical and ethical dilemmas. On the practical side, using lay interviewers to collect diagnostic information has stirred controversy about the level of expertise necessary for this function. For example, the principal author of the $D S M-I I I$ stated that lay interviewers lack adequate training for rating the presence and severity of clinical symptoms, compromising accurate data collection and producing serious methodological flaws (Spitzer, 1984). Empirical work also suggests that lay interviewers lack the appropriate skills for gathering clinical data used in the formulation of research diagnoses. In studies that compare diagnoses obtained by lay interviewers with those formulated by professional clinicians, overall agreement, particularly for major depression, tends to be low (Anthony et al., 1985; Helzer et al., 1985; Wing, Henderson, \& Winkel, 1977).

Concerns about using survey strategies in psychiatric research typically focus on practical kinds of issues - the professional status of the interviewers, their ability to gather diagnostic information, and attendant methodological issues - to the neglect of ethical issues. Yet the ethical issues are also important.

Based on the authors' experience in a recent survey that examined married couples' responses to life stress, this paper addresses some of the issues that emerge when survey research methods are used to study psychiatric vulnerability in the general population. Specifically, we discuss the issues of respondent harm and informed consent that result from the collaboration of survey and clinical methodologies, and discuss related ethical issues. Some practical difficulties encountered when lay interviewers are employed for sensitive research studies are also discussed. Initial solutions and strategies for dealing with these problems are outlined.

\section{METHOD}

The study was conducted as part of the 1985 Detroit Area Study (DAS). The DAS began in 1951 under the auspices of the Department of Sociology at the University of Michigan. It is designed as a vehicle for scholars to collect data in an urban laboratory and to train doctoral students in survey research methodology. The topic of the study changes yearly. In 1985, the topic was responses to stressful life experiences and related psychopathology among married couples. A general population sample of 1,755 respondents in suburban Detroit participated in the study.

To discern both the type and the severity of psychopathology, a two-phase screening procedure was used, involving two stages of interviewing (McLeod, Turnbull, \& Kessler, in preparation). At the first stage, a screening instrument was administered by lay interviewers to identify respondents who were probably psychiatric cases. These respondents were interviewed again at the second stage by a mental health professional who gathered diagnostic information. Twostage procedures allow the assessment of 
psychiatric disorders among a large sample of persons, while avoiding the high costs of large-scale diagnostic interviewing. For purposes of this paper, the two-stage procedure offers another advantage: we can compare the practical and ethical issues raised by the two different types of interviews.

In this study, sociology graduate students and professional lay interviewers employed by the Survey Research Center of the Institute for Social Research interviewed the respondents at the first stage. Respondents were asked about stressful life events during the year before the interview and about recent psychological distress, among other topics. Both the measure of life events and the measure of psychological distress differed from those typically used in survey studies of life stress. Most studies present respondents with a long list of life events and ask whether any of them have occurred to the respondent. Here, the long list was divided into a series of short lists of events which were scattered throughout the questionnaire. We asked additional detailed questions about the most serious and complex events, to probe for their context and their date of occurrence. The probing questions allowed respondents to discuss thoroughly each event and its relationship to other events in their lives. They also afforded respondents an opportunity to ventilate their feelings about the event.

The Diagnostic Interview Schedule (DIS) (Robins, Helzer, Croughan, \& Ratcliffe, 1981) was used to measure psychological distress. The DIS differs from symptom screening scales, the usual measures of psychological distress, in that it is designed to be a hierarchically-structured, clinically useful instrument that renders unbiased psychiatric diagnoses. Furthermore, the DIS is superior both to symptom scales and to clinical interviews because it is designed to obtain clinical information but can be administered by lay interviewers instead of mental health professionals. The DIS was used as the first-stage screening instrument.

All respondents identified by the DIS as currently experiencing an episode of depression, were considered eligible for the stage two diagnostic interview. In addition, individuals who were not currently depressed, but who had experienced, within the past six months, an episode of depression lasting two weeks or more and during which they experienced any of a list of depressive symptoms, were considered eligible. We pursued stage two interviews with both members of any couple in which either member was eligible and both had participated in the stage one interview. Nonsymptomatic spouses of eligible respondents served as controls.

During the stage two interview, psychologists and psychiatric social workers with extensive experience in psychiatric diagnosis made a thorough assessment of symptoms of depression and anxiety using the Schedule for Affective Disorders and Schizophrenia (SADS) (Spitzer, Endicott, \& Robins, 1978). The SADS rates the presence and severity of symptoms of major psychiatric disorders, and then uses these ratings to formulate diagnoses according to the Research Diagnostic Criteria. In this study, only questions relevant to depression and generalized anxiety disorder diagnoses were included and only these diagnoses formulated.

The first and second stage interviews contained different types of questions, and each stage used interviewers with a different level of training and orientation to the interview. Consequently, it is not surprising that each stage raised different practical and ethical issues. The issue of respondent distress will be considered separately for each stage, ethical issues raised by respondent distress will be discussed, and implications of these experiences for future research efforts will be summarized.

\section{RESPONDENT DISTRESS}

\section{Stage One Interview}

We encountered problems with the stage one instrument during the first pretest, at which time the complete DIS depression 
and anxiety sections were included in the questionnaire. Lay interviewers were upset by the distress they observed among some pretest respondents, and they attributed this distress to what they saw as the unethical and provocative nature of the DIS questions. The interviewers also noted that the life event questions evoked strong emotional reactions among some respondents. We were frankly surprised at the interviewers' reactions, given that earlier studies using the DIS did not report similar problems. Nor had problems been reported in previous efforts to elicit detailed life event information from general population respondents. Nevertheless, we were concerned that our interview would routinely cause distress among both interviewers and respondents. Witness the response of the principal investigator to the lay interviewers' discomfort and attendant reactions.

The interviewers freaked out in the pretest. One came upon a suicidal woman who got stirred up by the interview. So I am cutting the DIS in the first interview. We will use a screening instrument and hope we get a good response rate for the Phase II interview. Interviewers are on the verge of refusing to do the DIS. They say it is a diagnosis and that respondents know it. The interviewers feel upset about doing this.

Pretest data collection was impeded by the lay interviewers and could have led to poor data collection in the future. Although we considered dropping the DIS from the questionnaire, doing so would have jeopardized the critical focus of the study. Furthermore, it was not clear from interviewers' descriptions of the problems they encountered whether their reactions reflected respondent distress or their own empathic distress at hearing about human suffering.

Believing that complete withdrawal of the DIS would be an overreaction, we implemented four alternative procedures in response to lay interviewer objections. Through these procedures, we hoped both to reassure the interviewers and to protect respondents from harm. First, a "bail-out" mechanism was created, consisting of a series of demographic questions which the lay interviewer could use to bring the interview to a close. No hard and fast rules governed its use. If troubled by respondent distress or convinced that the interview material was too emotionally laden for a particular respondent, the interviewer had the prerogative of turning to these factual questions as a means of objectifying the situation and assuaging respondent distress. Second, the mental health professionals hired as stage two interviewers doubled as clinical backups for the lay interviewers. They were available to provide service to respondents if and when the situation warranted. Third, a referral booklet that contained information about community services and agencies, including crisis and central referral telephone numbers, was routinely left with each respondent. Fourth, we added compensatory questions about positive experiences.

These changes proved very successful in minimizing respondent and interviewer distress. The impact of the stage one interview on respondents was assessed in several ways, beginning with the most extreme reactions. The most serious form of distress would presumably be indicated by use of the bail-out section of the questionnaire and of the clinical back-ups. The bail-out section of the questionnaire was never used during any of the 1,755 interviews; no situation arose in which the interviewer felt uncomfortable completing the questionnaire as written. Similarly, the lay interviewers called for clinical back-up only five times; in each case, the call was unrelated to the interview material $(e . g .$, a respondent's son needed psychiatric hospitalization and the respondent asked for help in dealing with the attendant trauma). These observations suggest that the interview questions did not cause extreme distress to respondents. It is possible, however, that respondents were affected in other, more subtle ways by the interview.

Interviewer observations of respondents' 
reactions provide clues about other effects of the interview experience on respondent functioning. Three questions answered by interviewers are particularly relevant: Was the respondent distressed by the interview? Did the respondent express relief for having talked? Did the respondent want to talk still more at the end of the interview?

Only $45(2.6 \%)$ of the 1,755 respondents were rated as being distressed by the interview. This compares to $108(6.2 \%)$ respondents who expressed relief for having talked and 243 (13.9\%) who wanted to keep talking at the end of the interview. On balance, more respondents enjoyed the opportunity to talk than were distressed by it, according to interviewer ratings. When we examined the interviewers' assessment of the reasons for the distress, we found that most respondent distress was attributed to one of three causes: anxiety about the spouse's reaction to the respondent's participation in the interview; the length of the interview; and particular questions that made the respondent uncomfortable. The first two causes are not study-specific, relating to the more general interview situation rather than the content of the questionnaire. The last cause, however, speaks directly to the content of the study questionnaire and should be examined more closely.

Interviewers rated $210(12 \%)$ respondents as having been made uncomfortable by some of the interview questions. The majority of the uncomfortable questions (53\%) were about recent events in the lives of the respondents. Some respondents found it difficult to elaborate about painful or embarrassing circumstances of the events. For example, one woman was overwhelmed during the interview by life event questions that prompted a reliving of her nephew's suicide. Another woman found it embarrassing to answer questions that revealed her husband's love affair. These respondents felt that the questions were too personal and demanded information they were not comfortable disclosing. At least as com- mon was the respondent who was not willing to disclose information about an event in the life of a loved one, e.g., a daughter's divorce. These respondents felt that they did not have any right to discuss the problems of their friends and relatives with a stranger. Under either of these circumstances, interviewers did not press for event information, but respected the respondent's right to refuse to answer the question.

The other questions most commonly reported as uncomfortable were about current income, educational attainment, the respondent's childhood home environment, and personality characteristics. These kinds of questions appear frequently in survey questionnaires and are usually assumed to be free of any emotional import. According to our interviewers' observations, that assumption may be incorrect.

One problem with the interviewer ratings we used in our assessment of respondent distress, however, is that they apply only to the time of the interview - we do not know how long the distress and unease persisted. Presumably, the amount of harm caused by the interview is directly related to the persistence of distress. Temporary distress or unease that dissipates by the end of the interview is of less concern than distress that persists for weeks. We have available one measure of behavior that is probably related to the long-term impact of the first interview - participation in the stage two interview.

If distress experienced during the first interview stage were related to agreeing to participate in the second interview, we would have grounds to believe that the first interview had a long-term impact on respondents. As it turns out, respondents who were rated as distressed by the first interview were only slightly less likely than those who were not distressed to participate in a second interview. The screening procedure in the first stage yielded 293 respondents who were eligible for the second. Of 22 "distressed" respondents who were eligible for the stage 
two interview, 12 refused to participate, nine granted the interview, and one had moved and could not be located. Thus, only a very small number of respondents were so affected by the interview that they refused to participate in further interviews. Even among these 12, the reasons for refusal are diverse, including concerns about the spouse's reaction $(N=4)$, not having enough time $(N=4)$, and refusing without explanation $(N=4)$. It seems, therefore, that the distress caused by the first interview was minor and short-lived.

\section{Stage Two Interview}

The questionnaire in stage two contained one section that rated the severity of symptoms associated with major depressive disorder and another section that did the same for generalized anxiety disorder. Discrete episodes of depression and anxiety, both current and those that had occurred during the past year, were assessed, as well as chronic dysphoria. Questions were asked to exclude diagnoses of alcoholism, drug abuse, schizophrenia, and antisocial personality disorder. In addition, diagnoses for mother, father, siblings, and children of the respondent were obtained, using the Family History Research Diagnostic Criteria ( $A n$ dreasen, Endicott, Spitzer, \& Winokur, 1977). Questions about personality and adolescence were also included.

In light of the lay interviewer reactions to the original DIS questions, we were concerned that the probing nature of the diagnostic questions in stage two would arouse unnecessary fears among respondents about their mental health status. We took three steps to alleviate respondent concerns. First, the closing statement for the stage one interview alerted respondents that they might be recontacted for a second interview. Second, the stage two interview opened with an assurance of confidentiality and a solicitation of questions about the first interview; this gave respondents an opportunity to express any concerns they might have.
Third, the clinical interviewers were instructed to do whatever seemed appropriate to restore the respondent's equilibrium in the face of expressed distress. Most of the clinicians reported that they were never called upon to use their clinical skills for this purpose and that respondents generally enjoyed the interview.

In order to assess the impact of the second interview on the respondents, we again asked interviewers to report whether the respondent was distressed by the interview, expressed relief for having talked, or wanted to talk still more, and whether or not any questions made the respondent uncomfortable. Of the entire sample of stage two respondents, including spouses $(N=353), 25$ (7.1\%) respondents were reported by the clinical interviewers to have been distressed by the interview experience; 47 $(13.3 \%)$ respondents were reported to have expressed relief for having talked; and 78 (22.1\%) respondents were reported as having wanted to talk still more at the end of the interview. As with the first interview, the second interview appears to have done more good than harm to its respondents. On the other hand, $106(30.0 \%)$ stage two respondents found some of the interview questions uncomfortable - a much higher percentage than in the first interview, although no specific section of the stage two questionnaire was cited as more uncomfortable than any other.

The most obvious interpretation is that respondents found the content of the second, diagnostic interview more distressing than the content of the first interview. A comparison of interviewer ratings from the first and second interviews supports this interpretation in that a higher percentage of respondents is reported to have been distressed by the second interview than by the first. Although a higher percentage of respondents is also rated as being relieved for having talked and wanting to talk more in the second interview (indicating that clinical interviewers were more likely than lay 
interviewers to rate respondents as having been affected in some way by the interview), a proportionately higher percentage of those who were affected were reported to have been affected negatively. This suggests that the contents of the second interview were more evocative than those of the first. In contrast, it is interesting to note that the clinical interviewers never expressed any qualms about the content of the interview. They reported verbally that respondents were very interested in the interview material and frequently expressed a preference for the second interview over the first.

\section{Reduction Strategies}

Regardless of interpretations of the lay interviewers' reactions, it is clear that some mild, short-term distress was observed among our study respondents. Feelings of agitation and frustration, apprehension about revealing sensitive problems, reduction in self-esteem, and other types of discomfort have been documented in psychological experiments (Humphreys, 1970; Milgram, 1963; Murray, 1980; Piliavin \& Piliavin, 1972). The possibility that survey interviews engender similar feelings makes intuitive sense. Acknowledging the potential harm inherent in survey studies of mental health is only half the battle; the other half is determining how to minimize it. We believe that the strategies we implementedproviding open-ended "ventilating" questions and offering respondents the option of skipping evocative questions-created an enjoyable interview experience for most respondents.

It must also be recognized, however, that some of our respondents experienced discomfort: despite our best intentions and efforts, they were distressed by the interviews. This being so, it seems likely that aspirations to prevent all discomfort are unrealistic and that some discomfort is inevitable in any survey study. If this is true, the mild distress caused by survey partici- pation must be balanced against the benefits that derive from survey research. The types of questions permissible in guaranteeing no discomfort are likely to be so bland as to be theoretically uninteresting. If respondent discomfort is a reality of survey research, we must consider options other than changing the types of questions we ask to ensure that ethical obligations are met. Several options exist. Among them are: 1) not initiating studies that are by definition unethical; 2) improving informed consent procedures; 3 ) considering the role of intervention in epidemiologic surveys; and 4) increasing training for interviewers.

\section{ETHICAL STANDARDS}

Whether certain studies are unethical because of possible traumatic impact on subjects and therefore should not be initiated is a question that can be argued from either a utilitarian or a deontological perspective. From a utilitarian perspective, if an end is worthwhile then reasonable means towards achieving that end are justifiable. From a deontological perspective, the means or the processes involved in achieving ends are so important that certain ends should be abandoned so as not to violate certain ethical precepts.

Epidemiologic studies should probably be approached from a cautious utilitarian position that is firmly centered on the principle of beneficence (Faden \& Beauchamp, 1986); that is, issues of respondent harm and welfare are of primary concern. In this frame of reference, a judgment must be made about the conflicting risks and benefits of the survey, and no study initiated where there appears to be substantial risks and insufficient benefits. Questions of what constitutes an ethically acceptable level of risk or discomfort to the subject must be balanced with the value of the study. A general guideline would be that the more sensitive and painful the information, the more important the purpose in obtaining it must be. 
Guidelines should be instituted to determine whether a study should be initiated at all. First, some material may be so sensitive that it should be elicited in a context other than a survey design so that adequate follow-up is ensured. Conversely, subjects should not be exposed to emotional stress in a survey unless the problem under study is significant and cannot be studied another way. Second, questions should not be asked where harmful outcomes cannot be monitored. Using the present study as an example, we would not survey married couples about issues that would unduly stir up conflict in their marriages unless there was an adequate mechanism in place to monitor conflict. When negative aftereffects are a possibility, a study should not be conducted unless respondents are fully informed of this possibility and volunteer nonetheless.

The need for follow-up can only be determined by pretesting an instrument, and it would be unethical to use an untested instrument where such monitoring would be unavailable. Serious aftereffects should be anticipated and mechanisms to handle them should be implemented in the event they occur. Further, it may be more effective to ask direct questions of respondents about their reactions at the end of the interview, rather than gather interviewer ratings. Similarly, subsamples of those who seemed distressed during the interview can be followed up to check for any aftereffects.

Finally, it also seems unethical to conduct a study or ask a question that cannot be kept confidential. There are some settings in which it may be impossible to ensure confidentiality, such as prisons or schools. There are also some topics where duty compels disclosure of the information. For example, in many states, mental health professionals are required by law to report cases of suspected child abuse or neglect to social and law enforcement authorities. There are at least two recent examples of prosecution for nonreporting which have bolstered the intent of this law (Zazakis, 1986). In our study, we were fortunate that no interviewers observed any evidence of abuse or neglect; if they had, our guarantees of confidentiality to research participants would have been in conflict with public health reporting laws. Social science researchers may need to draw on other disciplines, such as law and philosophy, in order to ascertain the optimal interface between respect for privacy and requirements for research on socially sensitive topics.

How potential risks are estimated in epidemiological studies is crucial. It is well and good for researchers to say that they will not conduct unethical studies, but unless those studies can be identified before they are carried out, such declarations become superficial.

\section{Informed Consent}

Guidelines for informed consent provisions are based on concerns for the autonomy of the respondent (Faden \& Beauchamp, 1986). Issues around the subject of informed consent reflect two significant values in our society: the dignity and integrity of the individual and the freedom of scientific inquiry. These values are often in opposition and in survey research the conflict is manifest in researcher's desire for a high response rate and the need to obtain informed consent.

Unanswered questions concerning concealment and deception are key to the issue of informed consent in community samples. While deception usually connotes outright lying to subjects, the practice of formulating psychiatric diagnoses without respondents' knowledge can be construed as a variant that is only slightly more honest. In psychological research, guidelines allow a study to be conducted without the subject's prior knowledge only when research objectives cannot be achieved without the concealment (American Psychological Association, 1971). In psychiatric epidemiological studies, full knowledge be- 
forehand reduces the likelihood that respondents will participate and increases the likelihood of biased data.

Thus, the investigator must follow certain guidelines and implement certain measures to deal adequately with the problems of informed consent, still get participation, and preserve the integrity of the study. First, respondents must be adequately informed of the aims, methods, anticipated benefits and potential risks and discomforts of the study. The consent process must contain evidence of reasonable efforts to explain that the interview actually is a mental health survey, that sensitive questions will come up, that participation is voluntary, and that the respondent can terminate the interview at any time and say no to any particular question. Second, concealment should be revealed after the interview and the subject then allowed to withdraw his or her data from the study when the concealment is revealed. Third, the investigator must take responsibility for assessing and dealing with stressful aftereffects. Fourth, an offer to answer inquiries should be made. Finally, respondents must be able to understand the contents of the consent form and lay interviewers must be able to establish whether someone is competent to give informed consent.

Epidemiologic surveys conducted under the auspices of medical schools have long been subject to stringent human use regulations that require full explanation of the study's purpose and the implications of consent, including potential harm and benefit rendered by participation, as well as an assurance of confidentiality (Association of American Medical Colleges, 1982). In these settings, informed consent requirements are considered only after a risk-benefit assessment has been made, and different levels of disclosure are considered appropriate for different forms of research (Faden \& Beauchamp, 1986). Distinctions have been made between research that acquires new knowledge in the course of providing care and is justified by its potential value for the sub- ject, and research that is defined as purely scientific, without value or purpose for the specific subject studied (Beecher, 1979). The former requires less disclosure, the latter more disclosure. Survey research falls into the latter category.

Since the potential for harm rendered by other social science disciplines is usually perceived as negligible in comparison to medical disciplines, most survey research settings still have consent policies that are inadequate for quasi-clinical investigations. For example, ethical concerns may be at the discretion of the project director or principal investigator rather than of independent ethics committees or institutional review boards. Survey researchers have tended to regard biomedical consent regulations as irrelevant impediments to participation, but the introduction of sensitive material into survey research forces reconsideration of this position. Survey investigators' fears about participation may be unfounded, since most procedures used to elicit consent do not have a negative effect on response rate, response quality, or respondents' evaluation of the interview; rather, they enhance respondents' favorable feelings towards the interview (Singer, 1978a, $1978 b)$.

\section{Intervention}

The inclusion of sensitive questions in epidemiologic studies raises questions about the appropriateness of clinical intervention. It is a complicated question and reasonable people differ about it. Currently, there is no consensus on the course of action for the researcher who becomes involved with distressed people who need help but have not asked for it. Our study provided clinical backup to lay interviewers and initiated referrals to appropriate community agencies only when requested. Other social epidemiologic research studies have been governed by a hands-off policy that views clinical intervention as an intrusion, prohibiting any clinical involvement (Bromet, Parkinson, 
Schulverg, Dunn, \& Gordek, 1982). The reasoning behind this policy is that intervention makes for biased responses and also violates the project's contract with the respondent. Regardless of the approach chosen for a particular project, routine intervention is an inappropriate role for data gatherers. As a rule of thumb, we recommend that no intervention be offered unless requested or in an emergency.

By emphasizing procedures for informed consent, we do not mean to ignore the problems of respondent distress. Warning respondents about the potential harm involved in the interview is no substitute for minimizing the risk. If social research continues to study stressful life events and related mental health outcomes in the effort to understand the relationship between stress and illness, the traditional survey research practice of employing professional lay interviewers to conduct interviews may need to be reevaluated. Mental health professionals possess skills that could be invaluable in survey studies of mental health, including knowledge of human behavior, a repertoire of interviewing techniques designed to establish a working relationship quickly and to master resistance, a better ability to assuage respondent concerns about their mental health status, and greater experience in observation and assessment. None of this, however, negates the fact that it costs more to employ mental health professionals than lay interviewers. These higher costs may make the cost of large surveys prohibitive unless funding agencies allow concomitantly higher budgets.

\section{Interviewer Training}

It is impossible to say which set of interviewers, lay or clinical, has a more accurate perception of nonpatient populations. However, we can surmise that the training and orientation brought by each group to the interview situation govern their responses. On the one hand, lay interviewers in our survey were clearly more af- fected than clinical interviewers by signs of distress. Lay interviewers may overreact and then find themselves in a quandary about ways to resolve the situation. This phenomenon has an indirect analogue in a study by Mendell and Rapport (1969), who examined factors behind professionals' decisions to hospitalize patients presenting for emergency psychiatric evaluations. They found that the likelihood of hospitalization was inversely related to the type of training and amount of experience possessed by the evaluator. These findings were interpreted as a reflection of the tendency for mental health professionals with less training to take more drastic interventive measures, probably as a function of uncertainty about their own competence in dealing with a situation.

A study by Wing and colleagues (1977) suggests an alternative interpretation for lay interviewers' reactions. Comparing the psychiatric ratings assigned to comparable sets of subjects by a nonclinical psychologist and a psychiatrist, they found that the psychologist assigned much higher scores on average. They attribute this finding to the different standards used by the two raters. Nonmedical interviewers compare subjects with their own concept of the normal, while psychiatrists compare subjects with severely ill patients. Although this interpretation differs from the interpretation put forward by Mendell and Rapport (1969) it proposes a similar overreaction of lay interviewers in assessing symptom severity.

On the other hand, it may be that the clinical interviewers accept respondent reactions as normal and react too casually to respondent distress because it compares favorably with the reactions to which they are accustomed in clinical settings. The clinical interviewers were amazed at how well adjusted many of the respondents seemed. There is another possibility: the empathic orientation that clinical interviewers brought to the encounter may have elicited greater respondent reactions. In an earlier general population study of psychological distress, 
respondents reported more symptoms to psychiatric interviewers than to lay interviewers, even when the psychiatrist's status was not openly revealed (Dohrenwend \& Shrout, 1981). The study investigators concluded that respondents were more open in the presence of psychiatric interviewers because of subtle indications by lay interviewers of their own unease with gathering the symptomatic material.

Given the problems in using mental health interviewers routinely, an alternative approach might be to design an expanded training program for lay interviewers. Lay interviewers are trained professionals who have worked well in traditional surveys. Moreover, lay people are routinely trained and utilized in crisis centers and for mental health volunteer positions. Such training would include knowledge of the elements of a crisis and normal human responses to aversive life events. This would equip lay interviewers to handle any intense affect that is evoked when important events and losses are probed.

\section{CONCLUSION}

Based on experience in a recent study, this paper has considered the issue of respondent harm introduced by the methodology of psychiatric epidemiology and accompanying ethical issues. The experience described here is not unique. Epidemiologic strategies are becoming routine, and present new challenges. Survey researchers are opened to involvement with distressed people and material is elicited that has the potential to invoke distress in both the well and the ill. Direct clinical involvement in epidemiological research may lend a perspective to researchers about the needs of respondents in their samples.

Educational efforts in both the academic and public domains can increase awareness about epidemiologic research and can also lead to better epidemiologic science. Ongoing educational programs about the nature of epidemiologic research and routine creation of reports to respondents may be beneficial. In academia, survey interviewing and epidemiologic research perspectives that include formal training in ethics should be incorporated into clinical curricula. Research ethics should be subjected to continued empirical analysis. Optimally, social epidemiologic research will strike a balance between the depth of the clinical world and the breadth of the survey world. The level and type of training required of survey interviewers warrants reevaluation. In addition, ethical policies approximating those of medical or experimental research need to be examined and reworded to fit the needs of survey research. These policies should be considered standard epidemiologic research practice.

\section{REFERENCES}

American Psychological Association. (1971). Ethical standards for psychological research. APA Monitor, 2(7), 9-28.

Andreasen, N., Endicott, J., Spitzer, R., \& Winokur, G. (1977). The family history method using diagnostic criteria. Archives of General Psychiatry, 34, 1229-1235.

Anthony, J.C., Folstein, M., Romanoski, A.J., Von Korff, M.R., Nestadt, G.R., Chahal, R., Merchant, A., Brown, C.H., Shapiro, S., Kramer, M., \& Gruenberg, E.M. (1985). Comparison of the lay Diagnostic Interview Schedule and a standardized psychiatric diagnosis. Archives of General Psychiatry, 42, 667-675.

Association of American Medical Colleges. (1982). The maintenance of high ethical standards in the conduct of research. Ad hoc committee on the Maintenance of High Ethical Standards in the Conduct of Research.

Beecher, H. (1979). Experimentation in man. Springfield, IL: Charles C Thomas.

Bromet, E., Parkinson, D., Schulverg, H., Dunn, L., \& Gordek, P. (1982). Mental health of residents near the Three Mile Island reactor: A comparative study of selected groups. Journal of Preventive Medicine, $1,225-276$.

Dohrenwend, B., \& Shrout, P. (1981). Toward the development of a two stage procedure for case identification and classification in psychiatric epidemiology. In R. Simmens (Ed.), Research in community and mental health (Vol. 2). Greenwich, CT: JAI Press.

Dunham, H. (1965). Community and schizophrenia: An epidemiological analysis. Detroit: Wayne State University Press.

Faden, R., \& Beauchamp, T. (1986). A history and 
theory of informed consent. New York: Oxford University Press.

Faris, R. \& Dunham, H. (1939). Mental disorders in urban areas. Chicago: University of Chicago Press.

Helzer, J.E., Robins, L.N., McEvoy, L.T., Spitznagel, E.L., Stoltzman, R.K., Farmer, A., \& Brockington. I.F. (1985), A comparison of clinical and Diagnostic Interview Schedule diagnoses: Physicians reexamination of lay-interviewed cases in the general population. Archives of General Psychiatry, 42, 657-666.

Humphreys, L. (1970). Tearoom trade: Impersonal sex in public places. Chicago: Aldine.

Last, J. (1983). A dictionary of epidemiology. New York: Oxford University Press.

McLeod, J., Tumbull, J., \& Kessler, R. (in preparation). Validation of the Diagnostic Interview Schedule and Schedule for Affective Disorders and Schizophrenia.

Mendell, W., \& Rapport, S. (1969). Determinants of the decision for psychiatric hospitalization. $A r$ chives of General Psychiatry, 20, 321-328.

Milgram, S. (1963). Behavioral study of obedience, Journal of Abnormal and Social Psychology, 67, 371-378.

Mischler, E., \& Scotch, N. (1963). Sociocultural factors in the epidemiology of schizophrenia, Psychiatry, 26, 315-353.

Murray, T. (1980). Learning to deceive. Hastings Center Report, 10, 1-16.

Piliavin, J., \& Piliavin, I. (1972). Effect of blood on reactions to a victim. Journal of Personality and Social Psychology, 23, 353-361.

Robins, L.N., Helzer, J.E., Croughan, J., \& Ratcliffe, K.S. (1981). National Institute of Mental Health Diagnostic Interview Schedule: Its history, characteristics, and validity. Archives of General Psychiatry, 38, 381-389.

Singer, E. (1978a). The effect of informed consent procedures on respondents' reactions to surveys. Journal of Consumer Research, 5, 49-57.

Singer, E. (1978b). Informed consent: Consequences for response rate and response quality in social surveys. American Saciological Review, 43, 144-162.

Spitzer, R. (1984). Are clinicians still necessary? Psychotherapy research: Where are we going and where should we go? New York: Guilford Press.

Spitzer, R.L., Endicott, J., \& Robins, M.D. (1978). Research diagnostic criteria: Rationale and reliability. Archives of General Psychiatry, 35, 773-782.

Tancredi, L. (1986). Ethical issues in epidemiologic research. New Brunswick, NJ: Rutgers University Press.

Wing, J., Henderson, A., \& Winkel, M. (1977). The rating of symptoms by a psychiatrist and a nonpsychiatrist: A study of patients referred from general practice. Psychological Medicine, 7, 713-715.

Zazakis, J. (1986). May a health care provider be held liable for damages as a result of failure to report suspected child abuse? Hospital CEO and Risk Managers' Newsletter, 3, 118-119. 